\title{
The rapid response system: an integrative review
}

John Rihari-Thomas ${ }^{a}$, Michelle DiGiacomo ${ }^{b}$, Phillip Newton ${ }^{c}$, David Sibbritt ${ }^{\mathrm{b}}$, Patricia M. Davidson $^{\mathrm{b}}{ }^{\mathrm{d}}$

aNursing Research Institute, Australian Catholic University \& St Vincent's Health Australia Sydney, 390 Victoria Street, Darlinghurst, NSW 2010, Australia

${ }^{\mathrm{b} F a c u l t y}$ of Health, University of Technology Sydney, PO Box 123, Ultimo, NSW 2007, Australia

'School of Nursing \& Midwifery, Western Sydney University, Locked Bag 1797, Penrith, NSW 2751, Australia

dSchool of Nursing, Johns Hopkins University, 525 North Wolfe Street, Baltimore, 21205, USA

\begin{abstract}
Background: Clinical deterioration and adverse events in hospitals is an increasing cause for concern. Rapid response systems have been widely implemented to identify deteriorating patients.
\end{abstract}

Aim: We aimed to examine the literature highlighting major historical trends leading to widespread adoption of rapid response systems, focussing on Australian issues and identifying future focus areas.

Method: Integrative literature review including published and grey literature

Results: Seventy-eight sources including journal articles and Australian government materials resulted. Pertinent themes were the increasing acuity and aging of the population, importance of hospital cultures, the emerging role of the consumer, and proliferation, evolution and standardisation of rapid response systems.

Discussion: Translating evidence to usual care practice is challenging and is strongly driven by local factors and political imperatives.

\section{Conclusion:}

Rapid response systems are complex interventions requiring consideration of contextual factors at all levels. Appropriate resources, skilled workforce and positive workplace cultures are needed for these systems to reach their full potential.

Impact Statement: The RRS model is evolving and adapting to practice models. Considering contextual factors and patient, provider and health system elements are crucial for ensuring intervention fidelity.

Keywords: Hospital rapid response team, clinical deterioration, emergency, emergency treatment 


\section{Introduction}

Internationally, there is widespread interest in developing rapid response systems (RRS) to identify individuals at risk of clinical deterioration (Shappell, Snyder, Edelson, Churpek, \& Investigators, 2018). Contemporary hospital wards are dangerous environments for patients due to the risk of adverse events and iatrogenic complications (Douglas, Robinson, \& Fahy, 2001; Ulrich, Zimring, Joseph, \& Choudhary, 2004; Wilson et al., 1995). This is particularly true as the population ages, resulting in greater numbers of elderly patients, many presenting with multiple co-morbidities (Pham et al., 2012).

Patients in the contemporary ward environment are now at higher risk of adverse clinical events than ever before as incidence of undetected deterioration and failure to escalate the deteriorating patient continue. From 2007-08 to 2011-12, Australian hospitalisations linked to adverse events increased from 4.8 to 5.3 per 100 (The Australian Institute of Health and Welfare, 2014). Today, intensive care, high dependency and coronary care units continue to house our sickest patients enabling more aggressive, invasive and extensive management regimens. What has changed over time is the acuity and complexity of the admitted general ward/unit patient in our acute care hospitals (Driscoll A, Currey J, Allen, George M, \& Davidson, 2014). Historical trends in the reduction of length of stay over the past few decades suggest that patients admitted to acute health facilities are generally of higher acuity, as 'well' patients are now discharged earlier and may have ongoing care regimes and follow up in community and outpatient settings (The Australian Institute of Health and Welfare, 2013). The number of separations for people aged 85 and over increased by $41 \%$ over the period $2006-07$ to $2010-11$, an average annual increase of $8.6 \%$ and most of these separations were for acute care (87\%) or rehabilitation care (8\%) (The Australian Institute of Health and Welfare, 2013).

This rising acuity of the general ward patient often occurs with increasing nurse to patient ratios and an increasingly diverse nursing skill mix. Interest in identifying deteriorating patients is driven by the need to reduce adverse events. (Winters \& Pham, 2011). System process failures leading to adverse clinical events have not only been identified in the research literature, but also in outcomes of numerous government commissions of enquiry. 


\section{Purpose}

The purpose of this paper was to examine the literature highlighting major historical trends in managing the deteriorating patient internationally. The guiding question of this review was to identify the state of the science, describe the socio-political context and identify drivers for model implementation within the Australian context.

\section{Method}

As data was sought from a diverse range of sources, the method of integrative review was chosen to allow synthesis of a wide range of concepts and themes pertaining to the evidence for implementation (Whittemore \& Knafl, 2005). In addition to scholarly research studies, the inclusion of guidelines, statistics, government documents and commissioned enquiries were essential in describing the development, evolution and justification of the RRS. The systematic review process was not chosen due to the requirement for content primarily consisting of experimental research and strict adherence to methodological process, limiting the scope of discussion of the paper (Moher, Liberati, Tetzlaff, \& Altman, 2010). Both the grey literature and published, peer reviewed data sources and the electronic data bases Medline, Embase, Cochrane database of systematic reviews, CINAHL and Pubmed were searched using the terms 'medical emergency team', 'rapid response system', ' rapid response team', 'deteriorating patients', 'early warning score' and 'Australia'. The chosen search period 1996 to present allowed for inclusion of seminal RRS studies. The initial search returned 119 articles (Figure 1). Sixty eight articles were rejected for either their direct irrelevance to the research topic or duplication. Inclusion of the remaining articles was based on the impact and importance of primary resources and research papers around patient deterioration in the Australian setting and their relevance to the emerging themes and discussions presented in the paper. Hand searching of retrieved material was also undertaken. Searches of the World Wide Web were conducted using Google and Google Scholar search engines to obtain further information including data from international, national and state government health sources. Articles were then reviewed and summarised according to model elements using the method of an integrative review. Elements were categorised by both chronology and/or theme to allow narrative and thematic synthesis. 


\section{Results}

The search strategy generated 76 sources, 51 journal articles were identified, 7 of which were systematic reviews. In addition, 21 Australian government sources were also identified consisting of 17 federal and state resources and four state initiated commissions of inquiry into health system failures. International policy and professional organisations were also included to provide a contextual backdrop particularly pertaining to evidence generation. These data were integrated and synthesised using a discursive method to provide a snapshot of contemporaneous issues allowing departure and exploration from the main topics to cover a varying range of subjects relating to RRS literature (Gale, 2010). The changing patient population, systemic growth, evolution and standardisation of implementation methods, influence of hospital cultures and the emerging role of the consumer in rapid response emerged as key themes from narrative analysis. As health care becomes increasingly politicized considering the deteriorating patient in the local context is important. Although international studies have been reviewed to identify evidence and trends in the RRS, this manuscript focuses on implications for the Australian health care system

\section{Rapid Response Systems}

Rapid response systems seek to implement systematic processes to prevent delays in recognising and responding to clinical deterioration (Hillman \& Braithwaite, 2014). RRS use the principles of early detection through predefined indicators of clinical deterioration and response. The most common terms given to clinicians who respond to rapid response activations include medical emergency teams (MET), rapid response teams, and critical care outreach teams (Salamonson, Heere, Everett, \& Davidson, 2006). Within acute settings, these teams are outsourced in a variety of ways, most typically from critical care units such as intensive care, coronary care or a mixture of both and usually consist of a senior medical officer and critical care nurse/s. Historically these teams were designated 'cardiac arrest teams' and were initiated when a patient was either pre or in an actual arrest state. These roles are now evolving to become decentralised from their 'home' critical care units, increasingly focusing on involvement in ward patient clinical management when signs of deterioration are first evident (Hillman, 2008; Hillman et al., 2005).

Winters and colleagues undertook a systematic review and meta-analysis of articles reporting outcomes of rapid response systems (Winters et al., 2007). Of the studies included in the review, 5 used historical controls, 1 concurrent controls, and 2 a cluster randomized design. The pooled relative risk for hospital mortality comparing 
rapid response teams to control was 0.76 (95\% confidence interval, $0.39-1.48$ ) between the two randomized studies and 0.87 ( $95 \%$ confidence interval, $0.73-1.04$ ) among the five observational studies. The pooled relative risk for cardiac arrest comparing rapid response systems to control was 0.94 (95\% confidence interval, $0.79-1.13)$ in the single randomized study and 0.70 (95\% confidence interval, $0.56-0.92)$ in four observational studies. The heterogeneity of study designs was an important consideration of this systematic review.

A further systematic review was undertaken by Chan (Chan, Jain, Nallmothu, Berg, \& Sasson, 2010). The review incorporated data on nearly 1.3 million hospital admissions. Implementation of a rapid response team in adults was associated with a $33.8 \%$ reduction in rates of cardiopulmonary arrest outside the intensive care unit (relative risk [RR], 0.66; 95\% confidence interval [Cl], 0.54-0.80) but was not associated with lower hospital mortality rates (RR, $0.96 ; 95 \% \mathrm{Cl}, 0.84-1.09)$. In a paediatric population, the use of rapid response teams was associated with a $37.7 \%$ reduction in rates of cardiopulmonary arrest outside the intensive care unit (ICU) (RR, $0.62 ; 95 \% \mathrm{Cl}, 0.46-0.84)$ and a $21.4 \%$ reduction in hospital mortality rates ( $\mathrm{RR}, 0.79 ; 95 \% \mathrm{Cl}, 0.63-0.98)$ (Chan et al., 2010). This review also found evidence that deaths were prevented out of proportion to reductions in cases of cardiopulmonary arrest, raising questions about mechanisms of improvement. In spite of this limited evidence for mortality reduction in many settings, rapid response systems became a standard of care throughout Australia. This expansion may have been led by the absence of conclusive evidence for mortality reduction being insufficient to discard the routine application of these systems.

Though reducing cardiac arrest rates is an extremely important indicator of RRS success, some argue that overall end point mortality rates may not be the most appropriate measure with which to analyse the effectiveness of this model (Hillman \& Braithwaite, 2014). Emerging data suggests that mortality depends more upon the nature of the patients underlying clinical state and type of interventions they receive rather than being a measure of hospital safety and effectiveness of rapid response systems (Hillman \& Braithwaite, 2014) (Shappell et al., 2018). More recent studies however have actually shown increasing trends to decreased mortality. Chen et al. (Chen et al., 2014) compared in-hospital cardiac arrest, in-hospital cardiac arrest related mortality and overall hospital mortality rates between a hospital with a mature rapid response system against three other similar centres without an existing formal system. While the overall mortality rate did not change for the hospital with a long standing rapid response 
system in place, there was a significant reduction in in-hospital cardiac arrest rates ( $50 \%$ lower), in-hospital cardiac arrest related mortality ( $40 \%$ lower) and lower overall hospital mortality (6\% lower) for the centres that previously did not have a rapid response system (Chen et al., 2014).

Results of RRS studies to date are still diverse and contentious despite their widespread adoption (Jones, DeVita, \& Bellomo, 2011; Ranji, Auerbach, \& Hurd, 2007). This can likely be explained by methodological variation within the clinical trials and the challenges of implementing complex interventions. However, in advancing the science of systems for RRS, it is also important to consider structural characteristics of various models and their adaptability to individual healthcare organisations. Poor resourcing, model design and implementation could impact on the uptake and long term success of the rapid response system.

\section{Rapid response criteria}

Track and trigger systems are formalised processes that employ periodic vital sign measures (track) with a predetermined action (trigger) when the measures are breached. (Australian Commission on Safety and Quality in Healthcare, 2009) The Australian Capital Territory and Northern Territory use numerical scoring with a Modified Early Warning Score (MEWS) system (Australian Capital Territory Health, 2009) to track clinical deterioration. The majority of other Australian states however employ vital sign parameter calling criteria as a track for initiating rapid response such as that used by NSW 'Between the Flags program (Clinical Excellence Commission, 2009a).

Hillman and colleagues (Hillman et al., 2005) investigating the antecedents to hospital deaths and the role of a medical emergency team in the 'MERIT' study began a series of primary research studies into acute inpatient deterioration that have led to Australia being a contemporary global leader in the field of model development in this area. Hillman's work around vital sign parameters for medical emergency teams began in the 1990's originating at Sydney's Liverpool Hospital resulting in the creation of calling criteria that is still current as the primary criteria for activating many Australian rapid response systems. In 2005 Jacques (Jacques, Harrison, McLaws, \& Kilborn, 2006) conducted a follow on study, 'signs of critical conditions and emergency responses (SOCCER), testing the efficacy of vital sign parameters established in the MERIT study in an attempt to further distinguish early from late signs of deterioration. This cross-sectional survey looked at 3,046 adult patients in five hospitals over a two week period. Of 
these patients, the existence of 26 early signs of deterioration (critical condition and adverse events) were found in $n=12,384$ instances and 21 late signs were identified in $n=1,410$ cases. Pulse oximetry was not part of the initial measures for calling criteria with early medical emergency team activation, but SOCCER found that decreasing saturations were evident in both early (SpO2 90-95\%) and late (SpO2 <90\%) signs with increased risk of death. Overall the study validated the original criteria but also showed the occurrence, and importance of earlier signs of deterioration.

\section{Subjective criterion}

The worried or concerned criterion is an essential element of any rapid response system (M. DeVita et al., 2010; Santiano et al., 2009). For many years the nursing literature has discussed nurses as having an intuitive feeling that 'something is just not quite right' or patients 'have that look about them' (Cioffl, 2008; King \& Appleton, 1997; McCutcheon \& Pincombe, 2001). The worried criterion taps into this subjective patient assessment, acknowledging that nurses also possess their own unique sets of assessment skills. This criterion also covers all assessment aspects and events not relating directly to objective vital sign parameter breaches. The criterion was designed to empower nurses to escalate a patient's care by requiring a medical response within a specified timeframe. The potential exists therefore, to also be a possible tool for inter professional manipulation if not utilised appropriately. The literature reports on barriers and facilitators to nurses adopting rapid response systems (Astroth, Woith, Stapleton, Degitz, \& Jenkins, 2013; Jones et al., 2006). This same literature however is scant when reporting on the possible existence of issues such as nurses manipulating their medical colleagues with 'threats' of initiating rapid response if they are not satisfied with current management plans. Future studies with a strong focus on the cultural use of these systems may help to determine the existence or absence of changing professional balance between medical officers and nurses as a result of working within these systems.

\section{Australian government commissions of enquiry and inquests}

The need to mandate RRS across all national health facilities stemmed from commissions of enquiry initiated several high profile incidents occurring within the Australian health system (Douglas et al., 2001; Garling, 2008; Queensland Government, 2011). High rates of preventable adverse events have raised the attention of health professionals and policy makers alike. In the Australian state of New South Wales (NSW), a sequence of well-publicised adverse clinical 
events and growing public disquiet led to the Special Commissions of Inquiry into acute care services in NSW public hospitals (Garling, 2008; Walker, 2004). The Garling Report (Garling, 2008) made 139 recommendations, many addressing increased protection of patients, commentary on the nursing role and an increased emphasis on clinical cares including models of care utilised in our contemporary acute care facilities. Many in hospital failures documented in the extant literature also emerged as findings in the Garling Report. One major recommendation led to the mandated introduction of RRS for managing deteriorating patients including clinician education in all NSW public hospitals (Garling, 2008). Despite widespread implementation the continued occurrence of reportable critical incidents relating to adverse clinical events in ward patients suggests there are still process failures within these RRS systems.

Non-clinician escalation models for the deteriorating patient in Australia have not been a concept previously leading to mass appeal or widespread adoption. Recently however, a strong move to incorporate consumer and family activation into our rapid response models has occurred. In the Australian state of Queensland, the Office of the State Coroner's inquest findings into the death of Ryan Charles Saunders, (Queensland Government, 2011) a child who died of toxic shock syndrome, was instrumental in mandating a state wide process within the Queensland health system allowing for consumer and family escalation of care (Queensland Health, 2014). The Clinical Excellence Commission in NSW is also working with acute public health facilities to integrate consumer and family activation into its 'Between the Flags' rapid response model through the 'REACH' program (Clinical Excellence Commission, 2012b). At national level, National Safety and Quality Health Standard (NSQHS) 'Standard 9: Recognising and Responding to Clinical Deterioration, element 9.9 Mechanisms in place for patient / family/carer to escalate care response' (Australian Commission on Safety and Quality in Healthcare, 2012) will require all Australian acute care facilities to adopt this initiative into their rapid response programs in order to meet requirements for national accreditation. The foundation has now been set by the NSQHS to mandate processes for empowering patients, families and carers to enable escalation of care.

Consumers are now generally experiencing a more integrated clinician, patient and family centred approach to the acute inpatient journey within the Australian healthcare setting. Led by initiatives such as the NSW Clinical Excellence Commission's 'In Safe Hands' program, (Clinical Excellence Commission, 2012a) local governance is being 
diverted to the grass roots ward level. This includes the involvement of the patient and family in the multidisciplinary bedside clinical handover, inviting patients and their families to become more involved in medical management plans and decision making, with great relevance to deterioration and end of life discussions and care (Clinical Excellence Commission, 2012b).

\section{Progression to large scale Rapid Response Programs in Australia}

For many years, the implementation of RRS was inconsistent with institutions across Australia designing and running their own programs. The late 2000's saw the development and implementation of large scale programs for the first time, encompassing multiple sites over vast geographical areas. Two differing foundation models created the scene that set momentum for large government initiated schemes to follow around the nation.

\section{Compass}

Both the Australian Capital and Northern Territories adopted territory wide programs labelled 'Compass', (Australian Capital Territory Health, 2008) a modified version of the early warning score system (EWS) used in the United Kingdom (Royal College of Physicians, 2015). The modified early warning score (MEWS) utilises numerical scores to calculate a patient's acuity based upon pre-determined vital sign parameters (Australian Capital Territory Health, 2009; Subbe, Kruger, Rutherford, \& Gimmel, 2001). Scores are allocated according to how far a patients vital signs are seen as being deranged from a pre-determined set of parameters. The Compass program is assisted by an online learning and a training manual.

\section{Between the Flags (BTF) and Patient with Acute Condition for Escalation (PACE)}

In 2009, NSW rolled out a state wide initiative developed by NSW Health's quality pillar (Clinical Excellence Commission), which saw the implementation of a major rapid response program labelled 'Between the Flags'

(Clinical Excellence Commission, 2009a). The aim, to deliver standardisation of one rapid response program across the entire state incorporating all public healthcare centres within a diverse range of clinical settings. The red and yellow zones (tiers) of the program reflect surf life saver flags at the beach whereby a patient who's clinical parameters sit between the 'flags' (i.e. a non-coloured zone) is considered to be safe, those outside of this safe zone 
are considered at increased risk of deterioration. The BTF design was primarily suited to the medical emergency team concept in acute centres, but is capable of being interchangeable with other rapid response models and can be modified to meet lesser resourced centres such as those found in rural and remote areas.

Although there was intent of a 'one model state', NSW actually housed two of the largest programs in Australia at the time of BTF introduction. Former NSW Health's South East Sydney Illawarra Area Health Service' ‘Patient with Acute Condition for Escalation' (PACE) program also co-existed (South East Sydney Illawarra Area Health Service, 2009). These two programs differed in their approach to rapid response model design. Most large acute sites using the BTF model utilised a first line rapid response/medical emergency team outsourced from a critical care unit while PACE utilised the patient's admitting medical team as first line response. Over time, PACE adopted the BTF vital signs chart and calling criteria, though most PACE centres still use these tools within their admitting team model.

\section{Heterogeneity of implementation models.}

Despite aiming for standardised RRS processes, model diversity still exists both between and within Australian states. Single and multi-tiered, aggregated scoring and vital sign parameter activation, critical care and non-critical care led first line response give rise to debate as to the effectiveness of one model over another.

Gao et al. conducted a systematic review of the reliability and validity of physiological track and trigger systems (Gao et al., 2007). They examined 36 papers containing 25 different systems. Outcome measures for all studies were similar, death, admission to a critical care unit or not for resuscitation orders. The investigators however found low sensitivity in these outcomes (median quartile 43.3) along with low predictive values (median quartile 36.7). It was conclusive that there was little evidence of reliability, validity or utility within the studies they examined. The low sensitivity was explained as possibly being due in part to the nature of the physiology monitored, or perhaps the threshold value of the trigger itself. The study could not identify one type of track and trigger system to be better than another. Another systematic review by Smith et al. looking at the performance of aggregate weighted track and trigger systems was also inconclusive, once again pointing out inconsistency around physiological components of the systems (Smith, Prytherch, \& Schmit, 2008). 
Scrutiny around the use of early warning score systems such as the modified system (MEWS) utilised in the ACT/NT also leaves some doubt to their effectiveness as the ultimate design for rapid response. Although utilised widely in the United Kingdom, scoring systems can be more complicated than simple observation parameter criteria, there is also potential for inaccurate calculation (Australian Capital Territory Health, 2009; McGaughey et al., 2007).

Comparative effectiveness of RRS and the impact each may have on patient clinical outcomes requires further exploration. Until it is determined which model, if any, is the most optimal in assisting with early recognition and prevention of further clinical deterioration, then it is left to government policy and personal preference to decide.

The primary aim of rapid response models that use a non-critical care unit based approach (such as the admitting medical team) is skill enhancement of both medical and nursing staff directly responsible for patients care. As first line, their responsibility is to not only detecting deterioration, but to assess and implement management plans themselves rather than handing this responsibility to an 'outside' medical emergency team in the first instance. Critical care unit based teams are generally unfamiliar with the ward patient and their history, nor do they routinely continue with direct care after the acute event has past. Evidence that these models do in fact increase the skills and knowledge of the patients own primary care clinicians does not however currently have enough weighted research to draw on a definitive conclusion to the argument. The possibility may exist that some clinicians do not acquire or increase their assessment skills within this type of model environment, which may lead to a 'fall back' to, and in some cases a possible delay in rapid response activation or escalation to a critical care team assessment.

Advocates of the medical emergency team (critical care unit) based approach would argue that as core business, critical care teams deal with unstable patients on a daily basis and are therefore best equipped with both the knowledge and skills to manage those who are deteriorating in the ward environment (Buist et al., 2002; DeVita et al., 2004). Identified as a disadvantage in the MERIT study in reference to the medical emergency team model, was the significant increase in the number of 'call outs' to the general wards/units that the team received as a consequence of ward patients breaching rapid response criteria. In today's landscape of tight clinical budgets, few critical care led teams would have the luxury of additional 'floating staff'. Significant logistical resource strain on their personnel most likely occurs when required to leave their own patients and units in order to attend those 
requiring assistance in the ward setting. Centres that use ICU liaison nurses or nurse led teams would face the same issues if not adequately resourced specifically for that purpose (Elliot, Chaboyer, Ernest, Doric, \& Endacott, 2012).

Jones et al. led a team of investigators looking into the composition and resourcing of rapid response teams in Australian hospitals (The ANZICS-CORE MET dose Investigators, 2011). Of the 39 sites studied, all had a 24 hour service but only $25 \%$ of these teams were funded, meaning that resources had to be taken away from other areas in order to operate the teams. The investigators also found significant variation in team composition. An interesting point was very few rapid response teams were led by an Admitting/Consultant Medical Officer, the majority were intensive care fellows/registrars with the most senior nurses coming from both intensive and coronary care units. As the changing role of the old cardiac arrest team continues to evolve, resourcing requirements will also need to shift away from a focus on advanced life support and the resuscitation team to that of early intervention and rapid response teams. In order to function effectively as early interceptors of clinical deterioration, they will need appropriate resourcing of staff, equipment and technology as well as tailored education and training.

\section{Standardisation}

Whilst the Australian Commission on Safety \& Quality in Healthcare (ACSQHC) released standardised national guideline recommendations for RRS, (Australian Commission on Safety and Quality in Healthcare, 2010) data collection and analysis around these systems remains inconsistent, both between and within our states and territories. Key performance indicators in NSW for example are reported from all acute facilities to the Ministry of Health on both cardiac arrest and rapid response rates. These data do not necessarily accurately reflect or compare true clinical activity, especially where differing rapid response models are being measured against the same KPIs. Medical emergency team facilities report their rapid response figures based on critical care team call out rates. Those facilities utilising admitting care team responder models only utilise the critical care based teams in their facilities if higher level escalation is required. Therefore, these types of models appear to be under activating \& reporting on their rapid response systems.

Cardiac arrest rates in Australian facilities are also a likely underestimation of actual figures, as areas such as emergency departments, operating theatres and intensive care units often manage their arrests 'in house'. Formal 
activation of a medical emergency team to these areas means arrest data may not be as accurately captured or reported in the entire hospital data.

Non-standardisation of definitions is also an issue of contention. Cardiac arrest for example is not standardised in facilities across Australia. Some report this by definition only as 'absence of cardiac output', reporting all other medical emergencies including respiratory only arrests separately. Others may include cardiorespiratory arrests under the same data set for reporting purposes. In 2012, the Australian Resuscitation Council changed the definition to '...combination of unresponsiveness and absent or abnormal breathing' (The Australian Resuscitation Council, 2010). Regardless of the measures implemented, contextual issues, such as patient case mix, staffing levels and clinician skill mix are not considered or cross referenced during analysis of these events, which may offer more accurate insight into real world system process failures and successes.

Elements of national standardisation are progressively occurring. The ACSQHC national consensus statement on essential elements for recognising and responding to clinical deterioration (The Australian Resuscitation Council, 2010) was released in 2011. Further, production of the Australian Council Healthcare Standards (ACHS) occurred in 2013. 'Standard 9: Recognising and Responding to Clinical Deterioration in the Acute Health Care Setting' (Australian Commission on Safety and Quality in Healthcare, 2012) assists all Australian facilities to achieve accreditation relating to deteriorating patient systems. ACSQHC and Queensland Health also developed and endorsed a 'national' vital signs chart (Hill, Horswill, Preece, \& Watson, 2010). Using a combination of vital sign parameters and numerical scoring, as well as human factor principles design is now being utilised in several Australian states.

\section{Education}

Implementation of educational strategies to increase clinician skills around deterioration and effectively support their roles within RRS has also occurred within the Australian healthcare setting (Australian Capital Territory Health, 2008; Clinical Excellence Commission, 2009b). Standard curriculum for most courses includes physical assessment concepts, detection, management and escalation of the deteriorating patient as well as communication and clinical 
handover strategies. A variety of media modalities are utilised in the delivery of these education programs. Hands on, high and low fidelity simulation is perhaps regarded as one of the most useful forms of educational delivery, although it's effectiveness as a teaching tool still requires further study (Harder, 2010). These practical programs are also often backed up with eLearning (Australian Capital Territory Health, 2008; Clinical Excellence Commission, 2009b). Clinical handover initiatives are also developing around Australia creating models to improve clinician communication. We are seeing strategies such as the return of bedside clinical handover with multidisciplinary clinician, patient and carer input to help plan better care around the patient's hospital journey (Clinical Excellence Commission, 2012a).

Despite an increasing emphasis placed on education to increase RRS success, it does not necessarily ensure understanding, acceptance, compliance or clinical practice change, nor does current research provide overwhelming evidence of its ability to improve critical thinking ability of bedside clinicians, assisting them to make better decisions faster. Although education contained relevant content and addressed specific issues identified in needs analyses, Fuhrmann et al. (Fuhrmann, Perner, Klausen, Østergaard, \& Lippert, 2009) concluded it did not influence clinician awareness of ward patients at risk, nor influence the outcomes of this patient group. Further analysis needs to be undertaken to study the effect of these education programs on changing clinical behaviour and the application of learnt skills.

The development of education specifically designed to teach awareness and clinical intervention of the deteriorating patient is still at the forefront of most RRS implementation plans. The multidisciplinary nature of many of these programs may have the added benefit of helping to improve team collaboration.

Despite the availability of education programs to enhance clinical skills, very little has been published to date on curricula to support actual roles of responders to rapid response. Responders must also take leadership roles, effectively communicate with other clinicians, and in many cases, alter the current management regimes set by the patient's own medical teams, crossing culturally instilled intra-professional boundaries. 


\section{Hospital Cultures}

Hospital cultures are complex, multifaceted and highly individualized. The degree of success or failure of RRS depends very much on a facilities adaptability and acceptance to change. It is not enough to simply introduce a rapid response system and expect it to work effectively and with full compliance. Van Der Weyden (Van Der Weyden, 2009) researched the attitudes of Australian clinicians to system change. Results showed that clinicians generally do value the implementation \& use of evidence based systems for client centred care within the context of the Australian health care setting. Looking more closely at cultural attitudes and behaviours within RRS, Salamonson and colleagues (Salamonson et al., 2006) identified nurse years of experience as a major factor in system activation. Experienced nurses believed the greatest benefits were getting immediate help or attention, followed by their use in early recognition and management of deterioration. Rapid response also provided a backup system if they were worried about a patient, or were not satisfied with a current medical management plan. From 2004 to 2006, the Robert Wood Johnson Foundation funded demonstration projects in nine geographic locations to support RRS (Foote, 2010). Focus group evaluation with key groups at one "robust" and one "late adopter" hospital in this evaluation provided important information about the characteristics of rapid response teams, and a view of these teams 'through the eyes of a nurse'. Their work provided new insight into what makes a rapid response team successful and underscores the importance of considering process issues as well as outcome issues in health system redesign. In 'robust' adopter centres where the teams were an accepted part of hospital system culture, nurses were confident they had a positive effect, and were activated without hesitation. The opposite seemed to be prevalent in more 'challenged centres', with nurses hesitant and more inclined to exhaust all other avenues before reluctantly activating a rapid response. Nurses were also worried about the attitude and level of assistance they would receive from rapid response team members on arrival in these later centres. Cultural leaders also played a part in the success of these teams in robust hospitals with both clear leadership and a 'no option' attitude to activation.

Azzopardi et al. examined attitudes and barriers to the medical emergency team in an Australian tertiary paediatric hospital (Azzopardi, Kinney, Moulden, \& Tibballs, 2011). Eighty percent of nurses and $45 \%$ of medical staff still preferred to contact the covering medical officer first before initiating a MET call. Jones et al. also reported that a 
similar pathway was also followed by activators in an Australian adult setting where $72 \%$ of nurses would call the covering medical officer before initiating MET (Jones et al., 2006; Jones et al., 2011).

There is currently limited information pertaining to the preference or confidence levels of clinicians initiating one type of rapid response model over another. Traditional roles of cardiac arrest teams may still be firmly embedded in the minds of some clinicians, causing hesitation and anxiety with activation. Comparing possible differences in levels of clinician comfort between activation of critical care versus admitting team models could be invaluable.

Workforce culture and flexibility in any clinical environment will determine acceptance and utilisation of new initiatives. RRS are clearly not a substitute for astute clinical judgement, monitoring and vigilance. It is also important that the views of a range of providers are considered when implementing these models. Cultural barriers need to be removed before effective system uptake can occur unimpeded. Contemporary acute health environments are extremely busy, often under staffed and under skilled, leaving clinicians at all levels experiencing multiple pressures. Rethinking our moral obligations to patients requires the removal, or at least reduction in both systemic and personal barriers. Professional egos have little place in RRS success where team effort, equality and communication are paramount for optimal patient care. Exploration of Inter-professional trust issues could further extract opinions of each profession by the other. Issues in communication are already present within the literature and exist between hierarchies within and between healthcare disciplines, significantly impacting on both intra and interdisciplinary communication in situations of patient deterioration, with possibilities of leading to delays in patient reviews and initiation of rapid response (Sutcliffe, Lewton, \& Rosenthal, 2004).

Changes in RRS models and trends, clinician skill mix and healthcare cultures may all impact on policy adherence and practice within healthcare facilities, shaping future research enquiry. In most Australian states, RRS are now governed by government initiated clinical emergency response system policies (Australian Commission on Safety and Quality in Healthcare, 2012; Clinical Excellence Commission, 2013). Despite policy development, evidence of non-adherence is underscoring the importance of examining implementation issues (Azzopardi et al., 2011; Jones et al., 2006; Jones et al., 2011). Lack of professional accountability may be a factor in non-enforcement of policy related practice (Wachter \& Pronovost, 2009). The NSW Ministry of Health, along with many other local, national 
and global health authorities are slowly emerging from years of operating under cultures of 'no blame'. Initially well intentioned, it may have inadvertently to a point, generated a sub culture where deficits in professional accountability by many health care clinicians occurs (Wachter \& Pronovost, 2009). Thinking around benefits in a degree of blame in medical culture and promoting reporting of non-complaint clinicians is supported by several authors (Australian Commission on Safety and Quality in Healthcare, 2010; Campbell \& Wilkin, 2001; The Joint Commission, 2008). Clinicians must take professional accountability, ownership and support of the RRS as a contemporary tool for keeping patients under their care safe.

Acceptance and uptake at clinical level is essential for success, but executive buy- in could prove an essential ingredient for added drive and leadership from the top. Clinical governance support helps promote acceptance, providing organisational solutions to barriers or issues that arise (Campbell \& Wilkin, 2001). At the ward level this same leadership should be driven by clinical champions/leaders. Utilisation of project / program coordinators may help in providing operational and educational support to clinicians, assisting with understanding, implementation, monitoring of compliance, collection and dissemination of data and reports and a valuable resource to escalate identified risks to executives. Loop closure, or feedback of rapid response activity and audit reports to clinicians at the local level is necessary for system engagement (Jamtvendt, Young, Kristoffersen, O'Brien, \& Oxman, 2006). Presenting and discussing relevant local data enables clinicians to analyse performance and raise awareness of issues that may otherwise have gone relatively unnoticed or not recognised as repeat occurrences. Feedback should have integration with hospital and government performance indicators and benchmarks for consistency of practice and reporting at all levels.

Utilisation of clinician support programs through mentoring and clinical supervision by senior clinicians could assist with leading a culture of acceptance. Junior medical officers may benefit greatly by increased clinical supervision, mentoring and teaching by admitting medical officers and other staff specialists. Similarly, junior nurses by more direct supervision and role modeling by their senior colleagues and managers (Clinical Excellence Commission, 2012a). 


\section{Future directions}

Today's acute care health facilities are high technology environments. These technologies are also becoming best practice in monitoring patient physiology for early signs of deterioration (Smith, Prytherch, Schmit, \& Featherstone, 2006; Tarassenko, Hann, \& Young, 2006). Smart monitors have the capacity to continuously collect and store vital sign information, urine output and neurological status. They also have ability to automatically escalate a patient by initiating RRS (Smith et al., 2006). Potential exists for this technology to bypass ward clinicians, making both cultural issues that impact on escalation, as well as missed opportunities to escalate due to poor clinical skill and decision making, inconsequential. Removing human elements of system obstruction is on one hand positive, it then completely removes important subjective clinical assessment of the patient prior to system activation occurring, leading to a possible waste of resources and clinician time. The impact of high rates of 'false positive' calls will need to be a focus of future studies as these automated systems become more widely accepted.

Trends are beginning to focus on reducing the frequency of unnecessary rapid response calls (Williams, CardonaMorrell, Stevens, Bey, \& Glasgow, 2017). Currently, high numbers of rapid response are seen as positive for the most part, demonstrating clinician engagement. Yet this same activation may suggest patients have already began their journey on the deterioration pathway, having been doing so for some time with haemodynamic compensation preventing early parameter breaches (Shappell et al., 2018). The RRS could perhaps then no longer be utilised as the predictor of the deteriorating patient, but rather an indicator that the patient had not been identified early enough as a potential risk. There is beginning discussion around development of systems and screening tools able to predict, upon admission, those who are more likely to require rapid response.

Questioning a patient's likelihood of increased risk, as well as the likelihood of recovery, presents itself as an ideal time point to consider end-of-life issues more closely for certain patient groups (Guy's and St Thomas', 2012) (Williams et al., 2017). Admitting medical teams are still for the most part, reluctant to have these conversations with their patients (Buss \& Sulmasy, 1998; Clinical Excellence Commission, 2012a). In many cases when a patient experiences an adverse clinical event or sudden irreversible deterioration, the role of delivering this conversation is often left up to the responders of rapid response. Too many patients and their families still experience the mental and physical trauma of a resuscitation, only for the patient to be documented 'not for cardiopulmonary 
resuscitation' in hindsight post event. In many cases this directive would have been appropriate prior to clinical adverse events, based on review and discussion of patient prognosis and co-morbidities. To date, there does not seem to be an educational model to train rapid response teams in dealing with end of life conversation (Nelson et al., 2015), despite being required to regularly take on this role. They suggest these programs should include core components of communication about benefits, risks, and alternatives, formulation of a rapid response plan and preferences for resuscitation, alleviation of symptoms, attention to immediate patient and family needs and the emotional needs of themselves and the clinicians responsible for patient care. Our hospitals are not equally staffed twenty four hours a day, it is therefore imperative that we develop and implement these types of curricula for rapid response teams to effectively manage the needs of those who are unlikely to recover from the deterioration trajectory.

In addition to education, clinicians can benefit from tools to assist and manage this patient group. Programs such as the 'AMBER Care Bundle' are being trialled in some NSW hospitals, providing guidance as to when and how to initiate conversations with patients and their families around choice of treatment deterioration occurs (Guy's and St Thomas', 2012). Having these conversations, leads to a more dignified and planned death process for both patients and clinicians. AMBER has shown to improve decision-making positively impacting on multi-disciplinary team communication. It has also shown increased nurse confidence in approaching medical colleagues to initiate discussion of treatment plans with patients and families. Early conversations avoid unnecessary use of the RRS and have also shown a remarkable reduction in emergency department readmission rates.

In summary, this review has pointed to several areas for future investigation if RRSs are to evolve and reach their full potential. Organisational culture, resourcing, the impact of technology on both clinicians and patient care and complimentary systems to reduce RRS activation will all impact on rapid response evolution and need to be analysed for their impact and improvement if our current systems are to surpass their current limitations (RihariThomas, DiGiacomo, Phillips, Newton, \& Davidson, 2017). This includes designing models that clinicians are comfortable in activating and appropriate to local socio-cultural context. The RRS, along with many other imposed systems, require clinicians to be prepared and supported, not just with initial implementation, but for ongoing sustainability. Positive and enabling cultures accepting of change are paramount if these systems are to be accepted 
(Rihari-Thomas et al., 2017).

For clinicians, using a RRS, resourcing also needs to be addressed. Evidence shows the ever growing trend into RRS for early intervention, yet resourcing still remains funnelled into resuscitation training and equipment. Resourcing of RRS Teams needs greater analysis in order to provide appropriate staffing/team mix and equipment (Rihari-Thomas et al., 2017). Further, the organisational cost savings that early intervention teams could bring through prevention of further deterioration of patients who, would otherwise, require either extensive resuscitation and / or transfers to expensive critical care units needs to be brought to the forefront of discussion.

The growth of technology is helping to identify patient vital sign parameter breaches, yet many systems exclude human assessment and interaction variables (M. A. DeVita et al., 2010). Inquiry into the impact these systems are generating on clinicians with consequent changes in interaction with other health workers as well as job satisfaction and culture should be studied for effect. In addition, there is a possibility clinicians may over time give up their assessment skills in favour of 'automated assessment' as a way of easing their workload. The impact of both end of life and improvement in the ability clinicians to have general resuscitation discussions as part of all patient's care planning, are approaches that could reduce the use of RRS, their resourcing, costs and inappropriate activation. Lastly, what is the real impact of the immense resources currently being injected into clinician education around RRS?

\section{Conclusion}

This review has highlighted contemporaneous trends in the implementation of the RRS, focussing on the Australian setting within a context of international evidence.

Dr Dana Edelson has said: "The answer to why rapid response teams haven't been more successful is a combination of two things. We don't call them often or early enough, and when we get there we don't always do the right thing." (Colwell, 2015). Future rapid response direction must include the elimination of negative human factors that impede their effectiveness, while promoting technology to help capture patients who might otherwise 'slip through the net'. Rapid response systems are complex interventions requiring consideration of contextual factors at local levels, 
reach their full potential.

\section{References}

Astroth, K., Woith, W., Stapleton, S., Degitz, R., \& Jenkins, S. (2013). Qualitative exploration of nurses' decisions to activate rapid response teams. Journal of Clinical Nursing, 22(19-20), 6. doi:10.1111/jocn.12067

Australian Capital Territory Health. (2008). Compass. Retrieved from http://www.health.act.gov.au/professionals/general-information/compass.

Australian Capital Territory Health. (2009). Policy; modified early warning scores. Canberra: ACT Health.

Australian Commission on Safety and Quality in Healthcare. (2009). Recognising and responding to clinical deterioration:use of observation charts to identify clinical deterioration. Sydney: Australian Commission on Safety and Quality in Healthcare.

Australian Commission on Safety and Quality in Healthcare. (2010). National consensus statement: Essential elements for recognising and responding to clinical deterioration. Sydney: The Australian commission on Safety and Quality in Healthcare.

Australian Commission on Safety and Quality in Healthcare. (2012). NSQHS standards. Standard 9: recognising and responding to clinical deterioration in acute health care. Ultimo.

Azzopardi, P., Kinney, S., Moulden, A., \& Tibballs, J. (2011). Attitudes and barriers to a medical emergency team system at a tertiary paediatric hospital. Resuscitation, 82(2), 167-174. doi:10.1016/j.resuscitation.2010.10.013

Buist, M., Moore, G., Bernard, S., Waxman, B., Anderson, J., \& Nguyen, T. (2002). Effects of a medical emergency team on reduction of incidence of and mortality from unexpected cardiac arrests in hospital: preliminary study. $B M J(324), 6$.

Buss, M., \& Sulmasy, D. (1998). The preparedness of students to discuss end-of-life issues with patients. Academic Medicine, 1998(73), 4.

Campbell, S., \& Wilkin, D. (2001). Primary care groups: Improving the quality of care through clinical governance. BJM, 322(7302), 1580-1582.

Chan, P., Jain, R., Nallmothu, B., Berg, R., \& Sasson, C. (2010). Rapid response teams: A systematic review and meta-analysis. Archives of Internal Medicine, 170(1), 18.

Chen, J., Ou, L., Hillman, K., Flabouris, A., Bellomo, R., Hollis, S., \& Assareh, H. (2014). The impact of implementing a rapid response system: A comparrison of cardiopulmonary arrests and mortality among four teaching hospitals in Australia. Resuscitation, 85(9), 1275-1281.

Cioffl, J. (2008). Heuristics, servants to intuition, in clinical decision making. Journal of Advanced Nursing, 26(1), 5.

Clinical Excellence Commission. (2009a). Between the flags. Retrieved from http://www.cec.health.nsw.gov.au/programs/between-the-flags

Clinical Excellence Commission. (2009b). Detecting deterioration, evaluation, treatment, escalation and communicating in teams (DETECT). Retrieved from http://www.nswhealth.moodle.com.au/DOH/DETECT/content. 
Clinical Excellence Commission. (2012a). In safe hands. Retrieved from http://www.cec.health.nsw.gov.au/programs/in-safe-hands

Clinical Excellence Commission. (2012b). Partnering with patients; REACH -patient and family activation. Retrieved from http://www.cec.health.nsw.gov/programs/partnering-withpatients

Clinical Excellence Commission. (2013). Recognition and management of patients who are clinically deteriorating. . Retrieved from http://www0.health.nsw.gov.au/policies/pd/2013/PD2013 049.html.

Colwell, J. (2015). Tweaking rapid response teams: Automation, new team members improving outcomes at some hospitals. ACP Hospitalist.

DeVita, M., Braithwaite, R., Mahidhara, R., Stuart, S., Foraida, M., \& Simmons, R. (2004). Use of medical emergency team responses to reduce hospital cardiopulmonary arrests. Quality and safety in health care, 13(4), 4.

DeVita, M., Smith, G., Adam, S., Adams-Pizarro, I., Buist, M., Bellomo, R., . . Winters, B. (2010). Identifying the hospitalised patient in crisis - A concensus conference on the afferent limb of rapid response systems. Resuscitation, 81(4), 7.

DeVita, M. A., Smith, G. B., Adam, S. K., Adams-Pizarro, I., Buist, M., Bellomo, R., ... Goldsmith, D. (2010). "Identifying the hospitalised patient in crisis"-a consensus conference on the afferent limb of rapid response systems. Resuscitation, 81(4), 375-382.

Douglas, N., Robinson, J., \& Fahy, K. (2001). Inquiry into obstetric and gynaecological services at King Edward Memorial Hospital 1990-2000. Perth: Government of Western Australia.

Driscoll A, Currey J, Allen, J., George M, \& Davidson, P. (2014). New cardiac models of care reduce patient access to specialist nurses: A Victorian cross-sectional pilot study. Australian Critical Care, 27(1), 17-27.

Elliot, S., Chaboyer, W., Ernest, D., Doric, A., \& Endacott, R. (2012). A national survey of Australian intensive care unit (ICU) liaison nurse (LN) services. Australian Critical Care, 25(4), 9.

Foote, S. (2010). Rapid response teams. The American Journal of Nursing, 110(9), 13.

Fuhrmann, L., Perner, A., Klausen, T., Østergaard, D., \& Lippert, A. (2009). The effect of multiprofessional education on the recogntiton and outcome of patients at risk on general wards. Resuscitation, 80(12), 3.

Gale, J. (2010). Discursive analysis: A research approach for studying the moment-to-moment construction of meaning in systemic practice. Human Systems: The Journal of Therapy, Consultation \& Training, 21(2), 7-37.

Gao, H., McDonnell, A., Harrison, D., Moore, T., Adam, S., Daly, K., ... Harvey, S. (2007). Systematic review and evaluation of physiological track and trigger warning systems for identifying atrisk patients on the ward. Intensive Care Medicine, 33(4), 667-679.

Garling, P. (2008). Final report of the special commission of enquiry: Acute care services in NSW public hospitals. Retrieved from Sydney:

Guy's and St Thomas'. (2012). The amber care bundle. Retrieved from www.ambercarebundle.org/documents/amber-care-bundle-a-guide-for-patients-theirrelatives-and-carer.pdf

Harder, B. (2010). Use of simulation in teaching and learning in health sciences: A systematic review Journal of Nursing Education, 49(5). doi:10.3928/01484834-20090828-08.

Hill, A., Horswill, M., Preece, M., \& Watson, M. (2010). The developmenet of the adult deterioration detection system (ADDS) chart. Sydney: Australian Commission on safety and Quality in Healthcare.

Hillman, K. (2008). Rapid response systems. Indian Journal of Critical Care Medicine: Peer-reviewed, official publication of the Indian Society of Critical Care Medicine, 12(2), 77.

Hillman, K., \& Braithwaite, J. (2014). Patient safety and rapid response systems. The Medical Journal of Australia, 201(11), 654-656.

Hillman, K., Chen, K., Creticos, M., Bellomo, R., Brown, D., Doig, G., . . Flabouris, A. (2005). MERIT study investigators, Introduction of the medical emergency team (MET) system: a cluster randomised controlled trial. Lancet, 365(9477), 6. 
Jacques, T., Harrison, G., McLaws, M., \& Kilborn, G. (2006). Signs of critical conditions and emergency responses (SOCCER): A model for predicting adverse events in the inpatient setting. Resuscitation, 69(2), 175-183.

Jamtvendt, G., Young, J., Kristoffersen, D., O'Brien, M., \& Oxman, A. (2006). Audit and feedback: Effects on professional practice and health care outcomes (review). The Cochrane Database of Systematic Reviews(2).

Jones, D., Baldwin, I., McIntyre, T., Story, D., Mercer, I., Miglic, A., ... Bellomo, R. (2006). Nurses' attitudes to a medical emergency team service in a teaching hospital. Quality and Safety in Healthcare, 15.

Jones, D., DeVita, M., \& Bellomo, R. (2011). Rapid-response teams. New England Journal of Medicine, 365(2), 139-146.

King, L., \& Appleton, J. (1997). Intuition: A critical review of the research and rhetoric. Journal of Advanced Nursing, 26(1), 8.

McCutcheon, H., \& Pincombe, J. (2001). Intuition: An important tool in the practice of nursing. Journal of Advanced Nursing, 35(3), 6.

McGaughey, J., Alderdice, F., Fowler, R., Kapila, A., Mayhew, A., \& Moutray, M. (2007). Outreach and early warning system (EWS) for the prevention of intensive care admission and death of critically ill adult patients on general hospital wards. Cochrane Database of Systematic Reviews, (3). Retrieved from doi:10.1002/14651858.CD005529.pub2.

Moher, D., Liberati, A., Tetzlaff, J., \& Altman, D. (2010). Preferred reporting items for systematic reviews and meta-analyses: The PRISMA statement. International Journal of Surgery, 8(5), 336341.

Nelson, J., Mathews, K., Weissman, D., Brasel, K., Campbell, M., Curtis, R., ... Lustbader, D. (2015). Integration of palliative care in the context of rapid response; A report from the improving palliative care in the ICU advisory board. Chest, 147(2), 560-569.

Pham, J., Aswanj, M., Rosen, M., Lee, H., Huddle, M., Weeks, K., \& Pronovost, P. (2012). Reducing medical errors and adverse events. Annual Review of Medicine, 63, 447-463.

Queensland Government. (2011). Inquest into the death of Ryan Charles Saunders. (COR 4449/07(0)). Brisbane: Office of the State Coroner Retrieved from 1.

www.courts.qld.gov.au/ data/assets/.../cif-saunders-rc-20111007.pdf.

Queensland Health. (2014). Ryan's Rule. Retrieved from https://www.health.qld.gov.au/cairns hinterland/html/ryan-home.asp.

Ranji, S., Auerbach, A., \& Hurd, C. (2007). Effects of rapid response systems on clinical outcomes: systematic review and meta analysis. Journal of Hospital Medicine., 2(6), 422-432.

Rihari-Thomas, J., DiGiacomo, M., Phillips, J., Newton, P., \& Davidson, P. M. (2017). Clinician perspectives of barriers to effective implementation of a Rapid Response System in an academic health centre: a focus group study. International journal of health policy and management, 6(8), 447.

Royal College of Physicians. (2015). National early warning score (NEWS): Standardising the assessment of actute-illness severity in the NHS. Retrieved from https://www.rcplondon.ac.uk/projects/outputs/national-early-warning-score-news

Salamonson, Y., Heere, B., Everett, B., \& Davidson, P. (2006). Voices from the floor: Nurses' perceptions of the medical emergency team. Intensive and Critical Care Nursing, 22(3), 138143.

Santiano, N., Young, L., Hillman, K., Parr, M., Jayasinghe, S., Baramy, L., ... Hanger, G. (2009). Analysis of medical emergency team calls comparive subjective to "objective" call criteria. Resuscitation, 80(1), 5.

Shappell, C., Snyder, A., Edelson, D. P., Churpek, M. M., \& Investigators, A. H. A. s. G. W. T. G.-R. (2018). Predictors of in-hospital mortality after rapid response team calls in a 274 hospital nationwide sample. Critical care medicine, 46(7), 1041-1048.

Smith, G., Prytherch, D., \& Schmit, P. (2008). Review and performance evaluation of aggregate weighted 'track and trigger' systems. Resuscitation, 77, 170-179. 
Smith, G., Prytherch, D., Schmit, P., \& Featherstone, P. (2006). Hospital-wide physiological surveillance - a new approach to the early identification and management of the sick patient Resuscitation, 71, 19-28.

South East Sydney Illawarra Area Health Service. (2009). Patient with acute condition for escalation (PACE): management of the deteriorating adult inpatient. Sydney: South East Sydney Illawarra Area Health Service.

Subbe, C., Kruger, M., Rutherford, P., \& Gimmel, L. (2001). Validation of a modified early warning score in medical admissions. QJM: an international journal of medicine, 94, 5.

Sutcliffe, K. M., Lewton, E., \& Rosenthal, M. M. (2004). Communication failures: an insidious contributor to medical mishaps. Academic Medicine, 79(2), 186-194.

Tarassenko, L., Hann, A., \& Young, D. (2006). Integrated monitoring and analysis for early warning of patient deterioration British Journal of Anaesthesia, 97(1), 64-68.

The ANZICS-CORE MET dose Investigators. (2011). Rapid response team composition, resourcing and calling criteria in Australia. Resuscitation, 83(2012), 563-567.

The Australian Institute of Health and Welfare. (2013). Australian hospital statistics 2011-12. Retrieved from Canberra:

The Australian Institute of Health and Welfare. (2014). Indicators of Australia's healh. (14). Canberra: Australian Government.

The Australian Resuscitation Council. (2010). Cardiac arrest-an introduction. Retrieved from www.resus.org.au

The Joint Commission. (2008). Behaviours that undermine a culture of safety. Retrieved from Oakbrook: http://www.jointcommission.org/assets/1/18/SEA 40.pdf

Ulrich, R., Zimring, C., Joseph, A., \& Choudhary, R. (2004). The role of the physical environment in the hospital of the 21st century: A once in a lifetime opportunity. Retrieved from Concord:

Van Der Weyden, M. (2009). In the wake of the Garling inquiry into NSW public hospitals: A change of cultures. The Medical Journal of Australia, 190(2), 51-52.

Wachter, R., \& Pronovost, P. (2009). Balancing "no blame" with accountability in patient safety. The New England Journal of Medicine, 361(14), 5.

Walker, B. (2004). Special commission of enquiry into Campbelltown and Camden Hospitals. Retrieved from Sydney:

Whittemore, R., \& Knafl, K. (2005). The integrative review: Updated methodology. Journal of Advanced Nursing, 35(5), 546-553.

Williams, M., Cardona-Morrell, M., Stevens, P., Bey, J., \& Glasgow, M. S. (2017). Timing of palliative care team referrals for inpatients receiving rapid response services: A retrospective pilot study in a US hospital. International journal of nursing studies, 75, 147-153.

Wilson, R., Runciman, W., Gibberd, R., Harrison, B., Newby, L., \& Hamilton, J. (1995). The quality in Australian health care study. The Medical Journal of Australia, 163(9), 458-471.

Winters, B., \& Pham, J. (2011). Rapid response sytems: A review of the evidence. In M. De Vita, R. Bellomo, \& K. Hillman (Eds.), Textbook of Rapid Response Systems (pp. 65-78). New York: Springer.

Winters, B., Pham, J., Hunt, E., Guallar, E., Berenholtz, S., \& Pronovost, P. (2007). Rapid response systems: A systematic review. Critical Care Medicine, 35(5), 1238-1243. 